like for any other 3R approach, acceptance in the European Union will not preclude full animal testing from being requested in other regions of the world.

In conclusion, while we agree that 3 Rs methods are needed for regulatory toxicity testing, it does not serve the cause to publish studies that give the impression to serve to replace, reduce and refine animal testing, but that do not stand up to scrutiny. Instead, scientific evidence and practicability should govern 3Rs activities.

\section{References}

Buesen, R., Oberholz, U., Sauer, U. G. and Landsiedel, R. (2016). Acute oral toxicity testing: Scientific evidence and practicability should govern Three Rs activities. Altern Lab Anim 44, 391-398.
ECHA - European Chemicals Agency (2016). Guidance on information requirements and chemical safety assessment. Section R.7.4 of Chapter R.7a, last update December 2016. https://echa.europa.eu/documents/10162/13632/information requirements $\mathrm{r} 7 \mathrm{a}$ en.pdf

Gissi, A., Louekari, K., Hoffstadt, L. et al. (2017). Alternative acute oral toxicity assessment under REACH based on subacute toxicity values. ALTEX 34, 353-361. doi:10.14573/ altex. 1609121

Schrage, A., Hempel, K., Schulz, M. et al. (2011). Refinement and reduction of acute oral toxicity testing: A critical review of the use of cytotoxicity data. Altern Lab Anim 39, 273-295.

\title{
Reply to Comment on "Alternative acute oral toxicity assessment under REACH based on sub-acute toxicity values"
}

\section{Andrea Gissi, Kimmo Louekari, Laurence Hoffstadt, Norbert Bornatowicz and Alberto M. Aparicio}

European Chemicals Agency (ECHA), Helsinki, Finland

e-mail:kimmo.louekari@echa.europa.eu

doi:10.14573/altex.1712011

First, we would like to point out that the purpose of our article (Gissi et al., 2017) is to clarify how a regulatory requirement can be adapted. In the REACH Regulation, there are many situations where the legal text formally mentions that an existing long-term test makes a short-term test unnecessary (e.g., subchronic studies can replace sub-acute studies, long-term aquatic toxicity can also cover short-term requirements), but nothing is mentioned for acute toxicity. With the analysis we presented, we have formalized how this could also be the case for acute toxicity. However, since the update of REACH legal text is a long process, the way to make such adaptations available to registrants before the $2018 \mathrm{REACH}$ deadline was the update of ECHA's REACH Guidance text (ECHA, 2016) based on the results described in this paper.

1. The estimates of the number of cases for which an acute toxicity study may not be available for the incoming REACH registrations are given in our article and are based on statistics derived from all registration data hosted by ECHA. We agree that the cases for which an acute toxicity study is missing are not very common. However, because of the high number of new registrations in 2018, even a relatively small proportion of those $(\sim 10 \%$, as explained in our article) would matter. Hence, to omit about 550 unnecessary animal tests with no scientific and regulatory value is worth exploring and communicating to the registrants. Furthermore, we ran a new analysis and found that about $24 \%$ of the substances that have a reliable and relevant sub-acute study provided, do not have an equivalently reliable and relevant acute oral toxicity study. For those substances, registrants have used weight of evidence (WoE) or other adaptations to cover the information requirement. With the update of ECHA's Guidance based on the results described in the article, registrants have one more possibility to use this adaptation.

2. It seems that Buesen et al. may have misunderstood the text in the Guidance and the article with regard to the testing strategy/ sequence. ECHA Guidance does not suggest that an acute toxicity study has to be available before a sub-acute toxicity study 
is performed. The wording of the Guidance refers to "existing data (... acute toxicity studies)", which is not the case we want the registrants to consider. It is obvious that for substances that already have both acute and subacute studies and for those with an acute oral study only, the approach that we suggest would not be applicable. We agree that the conventional order of testing is to start with an acute toxicity test and use the $\mathrm{LD}_{50}$ value/ estimate to select the appropriate dose levels of the sub-acute study. The testing sequence that we propose mainly concerns substances for which both acute and sub-acute toxicity studies are missing, and both are required (i.e., REACH Annex VIII and above). In that situation, a registrant can perform the subacute study first, and if low toxicity (NOAEL above $1000 \mathrm{mg}$ / $\mathrm{kg}$ ) is seen, the oral acute toxicity test becomes unnecessary, because a reliable prediction can be based on the results of the sub-acute toxicity study. Furthermore, we would like to point out that this testing sequence is clearly described in the revised Guidance (ECHA, 2016), Appendix R.7.4-1, as follows: "To use the WoE approach described below, the registrant should perform a sub-acute toxicity study before acute toxicity testing, and in case the test substance is shown to be of low toxicity, he should eventually use the results of the 28-day study to waive the acute oral toxicity study."

In case the acute oral toxicity study is not performed, the dose selection for the sub-acute toxicity study could be based on existing information, such as data on analogue substances, predictions based on structure or on dose-range finding studies. Therefore, our article does not contradict the Guidance. Since new approaches are needed to maximize the use of already available in vivo study results, we wanted to explore this possibility and found that it can be scientifically supported.

3. WoE requires more than one piece of information. Each piece of information needs to meet criteria on quality and relevance. We are aware of the limitations of the NRU assay. The NRU, according to the EURL ECVAM validation study, predicts low toxic substances well, whereas the prediction for toxic substances is generally poor. We mention in our article that the NRU assay is just one of several choices that the registrant has when building their WoE case. Other possible types of data are QSAR prediction, physico-chemical data (low reactivity and inertness), and low bioavailability.

Buesen et al. state that they are "unaware of definite guidance from the ECHA that specifies how correlations between the in vitro, acute toxicity, and repeated dose toxicity data should be established or which specific correlations would be acceptable for regulatory classification or non-classification." ECHA has not suggested or claimed any specific (quantitative) correlation between different data that can be used to cover acute oral toxicity. The regulatory approach/solution is WoE, as explained in the Guidance (ECHA, 2016) and in our article; this implies that consistent evidence of low toxicity from reliable data sources can be used by the REACH registrants to propose an adaptation of this information requirement. In dossier evaluation, ECHA will assess these adaptions and find whether the scientific evidence gives a sufficient level of confidence on the proposed prediction of the acute oral toxicity.

\section{References}

ECHA - European Chemicals Agency (2016). Guidance on information requirements and chemical safety assessment. Section R.7.4 of Chapter R.7a, last update December 2016. https:/echa.europa.eu/documents/10162/13632/information requirements_r7a_en.pdf

Gissi, A., Louekari, K., Hoffstadt, L. et al. (2017). Alternative acute oral toxicity assessment under REACH based on subacute toxicity values. ALTEX 34, 353-361. doi:10.14573/ altex.1609121 